\title{
SOME RESULTS ON EQUIVARIANT CONTACT GEOMETRY FOR PARTIAL FLAG VARIETIES
}

\author{
PETER CROOKS AND STEVEN RAYAN
}

\begin{abstract}
We study equivariant contact structures on complex projective varieties arising as partial flag varieties $G / P$, where $G$ is a connected, simply-connected complex simple group of type $A D E$ and $P$ is a parabolic subgroup. We prove a special case of the LeBrun-Salamon conjecture for partial flag varieties of these types. The result can be deduced from Boothby's classification of compact simply-connected complex contact manifolds with transitive action by contact automorphisms, but our proof is completely independent and relies on properties of G-equivariant vector bundles on G/P. A byproduct of our argument is a canonical, global description of the unique $\mathrm{SO}_{2 n}(\mathbb{C})$-invariant contact structure on the isotropic Grassmannian of 2-planes in $\mathbb{C}^{2 n}$.
\end{abstract}

\section{INTRODUCTION}

Fano varieties with complex contact structures have been studied enthusiastically over the last half century, in large part due to their distinguished position at the intersection of complex algebraic geometry and real differential geometry. A compact quaternionic Kähler manifold with positive curvature always supports an $S^{2}$-bundle - its twistor space - the total space of which is a Fano contact manifold. As presented in [9], the LeBrun-Salamon conjecture [12] posits that every Fano contact manifold with $b_{2}=1$ is a homogeneous variety, one that is isomorphic to the unique closed orbit $\mathbb{P}\left(\mathcal{O}_{\min }\right)$ in the projectivized (co)adjoint representation of some simple Lie group G. If the conjecture were true, then every compact quaternionic Kähler variety with positive curvature would necessarily be homogeneous, and so progress on the LeBrun-Salamon conjecture is crucial to resolving an outstanding geometric classification problem within Riemannian geometry. The work of Beauville [1] is the strongest evidence thus far for the validity of the conjecture. For multiple points of view on Fano contact varieties, including the minimal rational curves and Mori theory approaches, we refer the reader to [4, 7,8, 13, 14].

On another front, complex contact manifolds have been studied in the context of equivariant geometry. Most notably, Boothby [2, 3] gives a complete classification of those compact simply-connected complex contact manifolds which are acted upon transitively by their respective groups of contact automorphisms (the so-called homogeneous complex contact manifolds). He identifies each with $\mathbb{P}\left(\mathcal{O}_{\min }\right)$ for a suitable simple group $\mathrm{G}$.

1.1. Results. This article presents some results on equivariant contact geometry for partial flag varieties. Firstly, we give a self-contained proof of the following special case of the LeBrun-Salamon conjecture.

Theorem 1. Let $\mathrm{G}$ be a connected, simply-connected complex simple group of type $\mathrm{ADE}$, and let $\mathrm{X}$ be a partial flag variety of $\mathrm{G}$ with $\mathrm{b}_{2}(\mathrm{X})=1$. If $\mathrm{X}$ is endowed with a $\mathrm{G}$-invariant complex

2010 Mathematics Subject Classification. 32M10 (primary); 14J45, 14M15, 22F30, 53D10 (secondary). 
contact structure, then there exists a $\mathrm{G}$-equivariant isomorphism $\mathrm{X} \cong \mathbb{P}\left(\mathrm{O}_{\text {min }}\right)$ of contact varieties, where $\mathcal{O}_{\text {min }}$ is the minimal nilpotent orbit of $\mathrm{G}$.

While Theorem 1 is deducible from Boothby's work, our argument differs significantly from that offered in [2,3]. Our approach is instead based on a sequence of results concerning the geometry (both equivariant and non-equivariant) of partial flag varieties $\mathrm{G} / \mathrm{P}$, where $P$ is a parabolic subgroup. Specifically, we prove that a G-invariant corank-1 subbundle $\mathbf{E}$ of $\mathbf{T}_{\mathrm{G} / \mathrm{P}}$ is completely determined as such by the isomorphism class of the quotient line bundle $\mathbf{T}_{\mathrm{G} / \mathrm{P}} / \mathbf{E}$ (see Proposition 3). This leads us to prove Proposition 5, which describes the contact line bundle of a G-invariant contact structure on G/P in terms of the isomorphism between $\operatorname{Pic}(\mathrm{G} / \mathrm{P})$ and the group of 1-dimensional P-representations. Proposition 7 and Theorem 9 then combine to give us the desired G-equivariant contact variety isomorphism between $\mathrm{G} / \mathrm{P}$ and $\mathbb{P}\left(\mathcal{O}_{\min }\right)$.

Secondly, we offer a detailed description of the contact manifold in Theorem 1 when $G$ is of type $D_{n}$. This manifold is precisely the Grassmannian $\operatorname{Gr}_{B}\left(2, \mathbb{C}^{2 n}\right)$ of those 2-planes in $\mathbb{C}^{2 n}$ which are isotropic with respect to the complex-bilinear dot product. While there are descriptions of the $\mathrm{SO}_{2 \mathfrak{n}}(\mathbb{C})$-invariant contact distribution $\mathbf{E}$ on $\mathrm{Gr}_{\mathrm{B}}\left(2, \mathbb{C}^{2 \mathrm{n}}\right)$ appearing in the literature (e.g. [7]), ours is global and canonical. Indeed, we use the classical identification of the tangent bundle of the full Grassmannian $\operatorname{Gr}\left(2, \mathbb{C}^{2 \mathfrak{n}}\right)$ with $\operatorname{Hom}\left(\mathbf{F}, \mathcal{O}^{\oplus 2 \mathfrak{n}} / \mathbf{F}\right)$, where $\mathbf{F}$ is the tautological bundle on $\operatorname{Gr}\left(2, \mathbb{C}^{2 \mathfrak{n}}\right)$. We then present $\mathbf{E}$ explicitly as a subbundle of the pullback to $\operatorname{Gr}_{\mathrm{B}}\left(2, \mathbb{C}^{2 \mathrm{n}}\right)$ of $\operatorname{Hom}\left(\mathbf{F}, \mathcal{O}^{\oplus 2 \mathrm{n}} / \mathbf{F}\right)$.

Acknowledgements. We gratefully acknowledge the support provided by Lisa Jeffrey and John Scherk. We also thank Steven Lu for useful discussions. The first author was supported by NSERC CGS and OGS awards. During this work, the second author was supported by a University of Toronto at Scarborough Postdoctoral Fellowship.

\section{Review of Properties of FANo Contact Varieties}

Here, we review the salient features of complex contact varieties in general and Fano contact varieties in particular. Let $X$ be a smooth complex variety of complex dimension $2 n+1$ for some $n \geq 0$, and let $\iota: \mathbf{E} \hookrightarrow \mathbf{T}_{X}$ be a rank- $2 n$ holomorphic subbundle of the tangent bundle $\mathbf{T}_{X}$. We say that the pair $(X, \mathbf{E})$ is contact if, in the short exact sequence

$$
0 \longrightarrow \mathbf{E} \stackrel{\iota}{\longrightarrow} \mathbf{T}_{\mathbf{X}} \stackrel{\theta}{\longrightarrow} \mathbf{L} \longrightarrow 0
$$

induced by $\mathrm{l}$, the composition of the Lie bracket on sections of $\mathbf{T}_{X}$ with the quotient map $\theta$ is an L-twisted bilinear form that is nondegenerate along $\mathbf{E}$. In keeping with the literature, we call the subbundle $\mathbf{E}$ the contact distribution; the quotient $\mathbf{L}$, the contact line bundle of $(X, E)$. If there exists an $\mathbf{E} \rightarrow X$ for which the pair $(X, E)$ is contact, we say that $X$ admits a contact structure.

From now on, we also assume that $X$ is projective, that $b_{2}(X)=1$, and that $X$ admits a contact structure, the distribution of which is $\mathbf{E}$. Let $\mathbf{L}$ be the associated contact line bundle. We use $\mathbf{K}_{\mathrm{X}}$ and $\mathbf{K}_{\mathrm{X}}^{\vee}$, respectively, for the canonical and anticanonical line bundles of $X$. In this case, $X$ is Fano with $\operatorname{Pic}(X) \cong \mathbb{Z}$ and $\mathbf{K}_{X}^{\vee} \cong \mathbf{L}^{\otimes n+1}$. This characterization is a consequence of a theorem of Demailly (Cor. 3 in [5]), applied to an earlier result of Kebekus, Peternell, Sommese, Wiśniewski (Thm. 1.1 in [9]). 
There are of course exactly two possibilities: either the contact line bundle $\mathbf{L}$ is a generator of $\operatorname{Pic}(X)$ or it is not. If it is not, then $L$ is a holomorphic $(n+1)$-th root of $K_{X}^{\vee}$ and $\mathrm{L}$ itself has nontrivial roots (namely, a generator of $\operatorname{Pic}(X)$ ). In this case, $X$ must be $\mathbb{P}^{N}$ for some N, by the well-known Kobayashi-Ochiai characterization of complex projective space [10]. Hence, whenever $X$ is a projective Fano contact variety with $b_{2}=1$ that is not a projective space, then it must be that $\operatorname{Pic}(X)=\mathbb{Z} \cdot[\mathbf{L}]$.

Taking these observations together, we have that:

- $\mathbf{L}$ is ample (in particular, it is the ample generator of $\operatorname{Pic}(X)$ whenever $X ¥ \mathbb{P}^{N}$ ); and

- if $\mathbf{L}^{\prime}$ is any other contact line bundle on $X$, then there must exist a holomorphic vector bundle isomorphism $\mathbf{L} \cong \mathbf{L}^{\prime}$.

The second fact is true because $\operatorname{Pic}(X) \cong \mathbb{Z}$ and $\mathbf{L}$ and $\mathbf{L}^{\prime}$ are holomorphic roots of the same line bundle (and hence $\operatorname{deg} \mathbf{L}=\operatorname{deg} \mathbf{L}^{\prime}$ ).

\section{Partial Flag Varieties and Contact Structures}

3.1. Basic Setup. Now, we specialize to the case where $X$ arises as a partial flag variety for a simple Lie group $\mathrm{G}$.

To be precise, let $\mathrm{G}$ be a connected, simply-connected complex simple group with Lie algebra $\mathfrak{g}$. Fix a maximal torus $T \subseteq G$ with Lie algebra $\mathfrak{t} \subseteq \mathfrak{g}$. Let $X^{*}(T)$ denote the weight lattice, and let $\Delta \subseteq X^{*}(T)$ be the resulting collection of roots. Also, let $(\cdot, \cdot)$ be the standard inner product on $\operatorname{span}_{\mathbb{R}}(\Delta) \subseteq \mathfrak{t}^{*}$. Choose collections $\Pi \subseteq \Delta^{+}$of simple roots and positive roots, respectively. Since $\mathfrak{g}$ is simple, there exists a unique highest root $\lambda$ with respect to the partial order induced by the choice of $\Pi$. Let $B \subseteq G$ be the opposite Borel with respect to our choice of positive roots. After fixing a subset $S$ of $\Pi$, let $\Delta_{S}^{+}$be the set of those positive roots expressible as $\mathbb{Z}$-linear combinations of the roots in $S$. One then has the standard parabolic subgroup $P_{S}$ generated by $B$ and the root subgroups coming from the roots in $\Delta_{S}^{+}$. Hence, the Lie algebra of $P_{S}$ is precisely

$$
\mathfrak{p}_{S}=\mathfrak{b} \oplus \bigoplus_{\beta \in \Delta_{S}^{+}} \mathfrak{g}_{\beta},
$$

where $\mathfrak{b}$ is the Lie algebra of $B$. Finally, let $W=N_{G}(T) / T$ be the Weyl group, and let $W_{S}$ denote the subgroup of $W$ generated by the simple reflections $\left\{s_{\beta}: \beta \in \Pi \backslash S\right\}$. The quotient $G / P_{S}$ is not only a complex projective variety, but is also Fano - see, for instance, Thm. V.1.4 in [11].

We will make extensive use of vector bundles on $G / P_{S}$ arising from $P_{S}$-representations via the associated bundle construction. More explicitly, suppose that $\varphi: P_{S} \rightarrow G L(V)$ is a finite-dimensional complex $\mathrm{P}_{\mathrm{S}}$-representation. One has a free $\mathrm{P}_{\mathrm{S}}$-action on $\mathrm{G} \times \mathrm{V}$ defined by

$$
\mathrm{p} \cdot(\mathrm{g}, v)=\left(\mathrm{gp}^{-1}, \varphi(\mathrm{p}) v\right),
$$

$g \in G, v \in V, p \in P_{S}$, with quotient variety denoted $G \times{ }_{P_{S}} V$. Note that the action of $G$ on $G \times V$ given by left-multiplication in the first factor commutes with the $P_{S}$-action, so that $\mathrm{G} \times{ }_{P_{S}} V$ carries a residual G-action. The projection map

$$
\begin{gathered}
\pi: G \times \times_{P_{S}} V \rightarrow G / P_{S} \\
{[(g, v)] \mapsto[g]}
\end{gathered}
$$


then realizes $G \times{ }_{P_{S}} V$ as a G-equivariant holomorphic vector bundle over $G / P_{S}$, called the associated bundle for $\mathrm{V}$. In short, $\mathrm{G} \times \times_{P_{S}} \mathrm{~V}$ is the G-equivariant holomorphic vector bundle over $G / P_{S}$ with fibre over $[e] \in G / P_{S}$ isomorphic to $V$ as a representation of $P_{S}$.

We now recall three standard group isomorphisms of importance to our work. Firstly, since $H^{1}\left(G / P_{S}, \mathcal{O}_{G / P_{S}}\right)=0=H^{2}\left(G / P_{S}, \mathcal{O}_{G / P_{S}}\right)$ (see [15]), the exponential sequence gives rise to a group isomorphism

$$
\begin{aligned}
\operatorname{Pic}\left(G / P_{S}\right) & \rightarrow H^{2}\left(G / P_{S} ; \mathbb{Z}\right) \\
{[\mathbf{F}] } & \mapsto c_{1}(\mathbf{F}) .
\end{aligned}
$$

Secondly, recall that

$$
\begin{aligned}
\mathrm{X}^{*}(\mathrm{~T})^{\mathrm{W}_{\mathrm{S}}} & \rightarrow \mathrm{H}^{2}\left(\mathrm{G} / \mathrm{P}_{\mathrm{S}} ; \mathbb{Z}\right) \\
\beta & \mapsto \mathrm{c}_{1}(\mathcal{L}(\beta))
\end{aligned}
$$

is a group isomorphism, where $\mathcal{L}(\beta)$ is the associated line bundle on $G / P_{S}$ arising from the 1-dimensional $P_{S}$-representation of weight $\beta$. We therefore have a third isomorphism,

$$
\begin{aligned}
X^{*}(T)^{W_{S}} & \rightarrow \operatorname{Pic}\left(G / P_{S}\right) \\
\beta & \mapsto[\mathcal{L}(\beta)] .
\end{aligned}
$$

It will be prudent to recall a natural $\mathbb{Z}$-basis of the group $X^{*}(T)^{W_{s}}$. For $\beta \in \Pi$, let $h_{\beta} \in$ $\left[\mathfrak{g}_{\beta}, \mathfrak{g}_{-\beta}\right]$ be the corresponding simple coroot. Note that the $h_{\beta}$ form a basis of $\mathfrak{t}$ dual to the basis of fundamental weights $\omega_{\beta} \in X^{*}(T), \beta \in \Pi$.

Lemma 2. The group $X^{*}(T)^{W_{s}}$ has a $\mathbb{Z}$-basis of $\left\{\omega_{\beta}: \beta \in \Pi \backslash S\right\}$.

Proof. Note that $\delta \in X^{*}(T)$ belongs to $X^{*}(T)^{W_{s}}$ if and only if $\delta$ is fixed by each simple reflection $s_{\beta}, \beta \in S$. This holds if and only if $\delta$ is orthogonal to each simple root in $S$. The desired conclusion then follows from the fact that

$$
\delta=\sum_{\beta \in \Pi} \delta\left(h_{\beta}\right) \omega_{\beta}=\sum_{\beta \in \Pi} 2 \frac{(\delta, \beta)}{(\beta, \beta)} \omega_{\beta}
$$

is the expression of $\delta$ as a linear combination of the fundamental weights.

We conclude this section with a proposition that will be of use later.

Proposition 3. If $\mathbf{F}_{1}$ and $\mathbf{F}_{2}$ are $\mathrm{G}$-invariant corank-1 subbundles of $\mathbf{T}_{\mathrm{G} / \mathrm{P}_{\mathrm{S}}}$ and the quotients $\mathbf{T}_{\mathrm{G} / \mathrm{P}_{\mathrm{S}}} / \mathbf{F}_{1}$ and $\mathbf{T}_{\mathrm{G} / \mathrm{Ps}_{\mathrm{S}}} / \mathbf{F}_{2}$ are isomorphic as holomorphic line bundles, then $\mathbf{F}_{1}=\mathbf{F}_{2}$.

Proof. To begin, note that $\left(\mathbf{T}_{\mathrm{G} / \mathrm{P}_{\mathrm{S}}}\right)_{[\mathrm{e}]}$ is canonically isomorphic to $\mathfrak{g} / \mathfrak{p}_{\mathrm{S}}$ as a $\mathrm{P}_{\mathrm{S}}$-representation, so that

$$
\mathbf{T}_{\mathrm{G} / \mathrm{P}_{\mathrm{S}}} \cong \mathrm{G} \times \times_{\mathrm{P}_{\mathrm{S}}}\left(\mathfrak{g} / \mathfrak{p}_{\mathrm{S}}\right) .
$$

The isomorphism (2) restricts to isomorphisms

$$
F_{1} \cong G \times P_{\mathrm{S}} V_{1}
$$

and

$$
\mathbf{F}_{2} \cong \underset{4}{\mathrm{G}} \times \mathrm{P}_{\mathrm{S}} \mathrm{V}_{2}
$$


where $V_{1}$ and $V_{2}$ are codimension- $1 P_{S}$-subrepresentations of $\mathfrak{g} / \mathfrak{p}_{\mathrm{S}}$. Since each T-weight space of

$$
\mathfrak{g} / \mathfrak{p}_{\mathrm{S}}=\bigoplus_{\beta \in \Delta^{+} \backslash \Delta_{\mathrm{S}}^{+}} \mathfrak{g}_{\beta}
$$

is 1-dimensional, each of $V_{1}$ and $V_{2}$ is obtained by removing a single weight space from the sum (3). Let $\gamma_{1}, \gamma_{2} \in \Delta^{+}$be the weights discarded to obtain $V_{1}$ and $V_{2}$, respectively. We then have bundle isomorphisms

$$
\mathrm{T}_{\mathrm{G} / \mathrm{P}_{\mathrm{S}}} / \mathbf{F}_{1} \cong \mathrm{G} \times_{\mathrm{P}_{\mathrm{S}}}\left(\left(\mathfrak{g} / \mathfrak{p}_{\mathrm{S}}\right) / \mathrm{V}_{1}\right) \cong \mathcal{L}\left(\gamma_{1}\right)
$$

and

$$
\mathbf{T}_{\mathrm{G} / \mathrm{P}_{\mathrm{S}}} / \mathbf{F}_{2} \cong \mathrm{G} \times \mathrm{P}_{\mathrm{S}}\left(\left(\mathfrak{g} / \mathfrak{p}_{\mathrm{S}}\right) / \mathrm{V}_{2}\right) \cong \mathcal{L}\left(\gamma_{2}\right) .
$$

In particular, $\mathcal{L}\left(\gamma_{1}\right) \cong \mathcal{L}\left(\gamma_{2}\right)$ as holomorphic line bundles over $G / P_{S}$, so that $\gamma_{1}=\gamma_{2}$. Hence, $V_{1}=V_{2}$, implying that $F_{1}$ and $F_{2}$ identify with the same subbundle of $G \times{ }_{P_{S}}\left(\mathfrak{g} / \mathfrak{p}_{S}\right)$ under (2). This completes the proof.

In the case that $\mathbf{F}_{1}$ and $\mathbf{F}_{2}$ define contact structures, we have the following immediate

Corollary 4. If each of $\mathbf{F}_{1}$ and $\mathbf{F}_{2}$ is the distribution of a $\mathrm{G}$-invariant contact structure on $\mathrm{G} / \mathrm{P}_{\mathrm{S}}$ and $\operatorname{Pic}\left(\mathrm{G} / \mathrm{P}_{\mathrm{S}}\right) \cong \mathbb{Z}$, then $\mathbf{F}_{1}=\mathbf{F}_{2}$.

This is simply the result of combining Proposition 3 with the fact $G / P_{S}$ is Fano (and then applying the second observation listed at the end of Section 2 .

3.2. The Projectivization of the Minimal Nilpotent Orbit. The material in 3.1 facilitates a worthwhile discussion of $\mathbb{P}\left(\mathcal{O}_{\min }\right)$ and its G-invariant contact structure. To this end, recall that the nilpotent cone of $\mathfrak{g}$ is the closed subvariety

$$
\mathcal{N}=\{\xi \in \mathfrak{g}: \operatorname{ad}(\xi) \text { is nilpotent }\},
$$

where ad $: \mathfrak{g} \rightarrow \mathfrak{g l}(\mathfrak{g})$ is the adjoint representation of $\mathfrak{g}$. The nilpotent cone is G-invariant and consists of finitely many G-orbits, called nilpotent orbits. The set of nilpotent orbits is partially ordered according to the closure order, namely $\mathcal{O}_{1} \leq \mathcal{O}_{2}$ if and only if $\mathcal{O}_{1} \subseteq \overline{\mathcal{O}_{2}}$. The non-zero nilpotent orbits have a unique minimal element, $\mathcal{O}_{\min }$, called the minimal nilpotent orbit. It is known that $\mathcal{O}_{\min }$ is the G-orbit of a non-zero vector in the lowest root space $\mathfrak{g}_{-\lambda}$.

Nilpotent orbits are invariant under scaling action of $\mathbb{C}^{*}$ on $\mathfrak{g}$. In particular, we have an inclusion of $\mathbb{P}\left(\mathcal{O}_{\text {min }}\right):=\mathcal{O}_{\text {min }} / \mathbb{C}^{*}$ into $\mathbb{P}(\mathfrak{g})$ as a closed G-orbit. Now, suppose that $\xi \in \mathfrak{g}_{-\lambda} \backslash$ $\{0\}$, which determines a class $[\xi] \in \mathbb{P}\left(\mathcal{O}_{\min }\right)$. The $\mathrm{G}$-stabilizer of $[\xi]$ is the standard parabolic subgroup $P_{\Lambda}$, where $\Lambda$ is the collection of those simple roots which are orthogonal to $\lambda$. We therefore have the G-variety isomorphism

$$
\begin{gathered}
\varphi: \mathrm{G} / \mathrm{P}_{\wedge} \stackrel{\cong}{\rightrightarrows} \mathbb{P}\left(\mathcal{O}_{\min }\right) \\
{[\mathrm{g}] \mapsto[\operatorname{Ad}(\mathrm{g})(\xi)] .}
\end{gathered}
$$

It turns out that $\mathbb{P}\left(\mathcal{O}_{\text {min }}\right)$ carries a distinguished G-invariant contact structure, $\mathbf{E}_{\text {min }} \subseteq$ $\mathrm{T}_{\mathbb{P}\left(\mathcal{O}_{\min }\right)}$. To obtain it, note that the Killing form on $\mathfrak{g}$ restricts to a G-equivariant variety isomorphism between $\mathcal{O}_{\min }$ and a coadjoint orbit in $\mathfrak{g}^{*}$. The latter has the Kirillov-KostantSouriau symplectic structure, so that $\mathcal{O}_{\min }$ is symplectic. The symplectic form on $\mathcal{O}_{\min }$ has 
weight 1 with respect to the $\mathbb{C}^{*}$-action, and Lemma 1.4 of [1] then gives the desired contact structure on $\mathbb{P}\left(\mathcal{O}_{\min }\right)$.

Using Remark 2.3 from [1], one can more explicitly describe the bundle $\mathbf{E}_{\min }$. Let $[\xi] \in$ $\mathbb{P}\left(\mathcal{O}_{\text {min }}\right)$ be the class of a lowest root vector, as above. Via the isomorphism (4) $\left(\mathbf{E}_{\min }\right)_{[\xi]}$ identifies with a codimension-1 subspace of $\mathfrak{g} / \mathfrak{p}_{\Lambda}$, the tangent space of $G / P_{\wedge}$ at the identity coset. Now, note that $\mathfrak{p}_{\wedge} \subseteq\left(\mathrm{g}_{-\lambda}\right)^{\perp}$, the orthogonal complement of $\mathfrak{g}_{-\lambda}$ with respect to the Killing form. Our fibre is then given by

$$
\left(\mathbf{E}_{\text {min }}\right)_{[\xi]}=\left(\mathfrak{g}_{-\lambda}\right)^{\perp} / \mathfrak{p}_{\Lambda} .
$$

Since $\mathbf{E}_{\min }$ is a G-invariant subbundle of $\mathrm{T}_{\mathbb{P}\left(\mathcal{O}_{\min }\right)}$, (5) can be used to determine the fibre of $\mathbf{E}_{\min }$ over any point.

3.3. Reduction to the Case of a Maximal Parabolic. Let us begin to directly address the classification of partial flag varieties admitting G-invariant contact structures. To this end, assume $S \subseteq \Pi$ is such that $\mathrm{G} / \mathrm{P}_{\mathrm{S}}$ admits a $\mathrm{G}$-invariant contact structure $\mathbf{E} \subseteq \mathbf{T}_{\mathrm{G} / \mathrm{P}_{\mathrm{S}}}$. In light of earlier remarks, we shall also assume that $b_{2}\left(G / P_{S}\right)=1$. This second assumption imposes a significant constraint on the subsets $S$ under consideration. Indeed, one has a Schubert cell decomposition of $\mathrm{G} / \mathrm{P}_{\mathrm{S}}$ into B-orbits,

$$
G / P_{S}=\coprod_{[w] \in W / w_{s}} B w P_{S} / P_{S},
$$

so that $\mathrm{H}^{2}\left(\mathrm{G} / \mathrm{P}_{S} ; \mathbb{Z}\right)$ is free of rank equal to the number of (complex) codimension-1 Schubert cells. Since the codimension of $B w P_{S} / P_{S}$ in $G / P_{S}$ is the length of a minimal-length coset representative in $[w] \in W / W_{S}$, the codimension-1 Schubert cells are those of the form $\mathrm{Bs}_{\beta} \mathrm{P}_{S} / \mathrm{P}_{S}, \beta \in \Pi \backslash S$. Hence, the condition $b_{2}\left(G / P_{S}\right)=1$ implies that $\Pi \backslash S$ has cardinality 1 , so that $S=\Pi \backslash\{\alpha\}$ for some unique $\alpha \in \Pi$. In other words, $P_{S}$ is a maximal parabolic subgroup of $\mathrm{G}$.

3.4. The Contact Line Bundle on G/Ps. We now give a more explicit description of the Ginvariant contact structure $\mathbf{E}$. Using the bundle isomorphism (2), we will regard the fibre $\mathbf{E}_{[e]}$ as a codimension-1 $P_{S}$-subrepresentation of $\mathfrak{g} / \mathfrak{p}_{\mathrm{S}}$. Of course, since $\mathbf{E}$ is a G-invariant subbundle of $\mathbf{T}_{\mathrm{G} / \mathrm{P}_{\mathrm{S}}}$, we also have

$$
\mathrm{E} \cong \mathrm{G} \times \times_{P_{S}} \mathbf{E}_{[e]} .
$$

Hence, the contact line bundle $\mathbf{L}=\mathbf{T}_{\mathrm{G} / \mathrm{P}_{\mathrm{S}}} / \mathbf{E}$ is given by

$$
\mathbf{L} \cong \mathrm{G} \times \times_{\mathrm{P}_{\mathrm{S}}}\left(\left(\mathfrak{g} / \mathfrak{p}_{\mathrm{S}}\right) / \mathbf{E}_{[e]}\right) .
$$

Using (7), one can describe $L$ in terms of the isomorphism $X^{*}(T)^{W_{S}} \cong \operatorname{Pic}\left(G / P_{S}\right)$.

Proposition 5. If $\mathfrak{g}$ is simply-laced, then the highest root $\lambda$ belongs to $X^{*}(T)^{W_{s}}$ and $\mathbf{L}$ is isomorphic to $\mathcal{L}(\lambda)$.

Proof. We begin with two observations. Firstly, we have $\mathbf{L} \cong \mathcal{L}(\gamma)$ for some $\gamma \in X^{*}(T)^{W_{s}}$. Secondly, since $\mathfrak{g}$ is simply-laced, $\lambda$ is the unique dominant root. Proving the proposition will therefore amount to showing that $\gamma \in \Delta$, and that $\gamma$ is dominant. For the former, note that each T-weight of $\mathfrak{g} / \mathfrak{p}_{\mathrm{S}}$ is a root. It follows that the weight of the quotient representation $\left(\mathfrak{g} / \mathfrak{p}_{\mathrm{S}}\right) / \mathrm{E}_{[e]}$ is also a root. Also, the isomorphisms (7) and $\mathrm{L} \cong \mathcal{L}(\gamma)$ together imply that $\gamma$ is the weight of $\mathfrak{g} / \mathfrak{p}_{\mathrm{s}}$, so that $\gamma$ must be a root. 
To prove that $\gamma$ is dominant, we note that

$$
\mathcal{L}((n+1) \gamma) \cong \mathbf{L}^{\otimes(n+1)} \cong \mathbf{K}_{\mathrm{G} / \mathrm{P}_{S}}^{\vee},
$$

where $2 n+1$ is the (complex) dimension of $G / P_{S}$. Also, the isomorphism (2) yields

$$
\mathbf{K}_{\mathrm{G} / \mathrm{P}_{\mathrm{S}}}^{\vee}=\wedge^{2 \mathfrak{n}+1} \mathbf{T}_{\mathrm{G} / \mathrm{P}_{\mathrm{S}}} \cong \mathrm{G} \times \times_{\mathrm{P}_{\mathrm{S}}}\left(\wedge^{2 \mathfrak{n}+1}\left(\mathfrak{g} / \mathfrak{p}_{\mathrm{S}}\right)\right) .
$$

By (3), the weight of $\wedge^{2 \mathfrak{n}+1}\left(\mathfrak{g} / \mathfrak{p}_{S}\right)$ is

$$
\mu_{S}:=\sum_{\beta \in \Delta^{+} \backslash \Delta_{S}^{+}} \beta,
$$

and we therefore have

$$
\mathbf{K}_{\mathrm{G} / \mathrm{P}_{\mathrm{S}}}^{\vee} \cong \mathcal{L}\left(\mu_{\mathrm{S}}\right) .
$$

Combining (8) and (9), we conclude that $(n+1) \gamma=\mu_{S}$. Since $\mu_{S}$ is dominant, this implies that $\gamma$ is dominant.

Before proceeding to the next section, we note that our arguments allow us to quickly recover the following well-known fact.

Corollary 6. The subbundle $\mathbf{E}_{\min } \subseteq \mathbf{T}_{\mathbb{P}\left(\mathcal{O}_{\min }\right)}$ is the unique G-invariant contact structure on $\mathbb{P}\left(\mathcal{O}_{\text {min }}\right)$.

Proof. Suppose that $\mathbf{F} \subseteq \mathbf{T}_{\mathbb{P}\left(\mathcal{O}_{\min }\right)}$ is a G-invariant contact structure. Let us first assume $\mathrm{G}$ to be of type ADE (so that $\mathfrak{g}$ is simply-laced). Note that both $\mathbf{E}_{\min }$ and $\mathbf{F}$ pull-back to G-invariant contact structures on $\mathrm{G} / \mathrm{P}_{\wedge}$ under the isomorphism (4). By Proposition [5, it follows that both contact line bundles $\mathbf{T}_{\mathbb{P}\left(\mathcal{O}_{\min }\right)} / \mathbf{E}_{\min }$ and $\mathbf{T}_{\mathbb{P}\left(\mathcal{O}_{\min }\right)} / \mathbf{F}$ pull-back to $\mathcal{L}(\lambda)$ under (4). In particular, these bundles are isomorphic, and Proposition 3 then implies $\mathbf{F}=\mathbf{E}_{\min }$.

If $\mathrm{G}$ is not of type $\mathrm{ADE}$, then $\operatorname{Pic}\left(\mathbb{P}\left(\mathcal{O}_{\min }\right)\right) \cong \mathbb{Z}$. Now, it follows from Corollary 4 that $\mathbf{F}=\mathbf{E}_{\text {min }}$.

\section{A Classification of G-Invariant Contact Structures on G/P}

4.1. The Main Theorem. We now consolidate the results presented in Sections 3.3 and 3.4. In light of Proposition 5, we will assume $G$ to be of type ADE for the duration of this article. We then have the following relationship between the simple root $\alpha$ from 3.3 and the highest root $\lambda$.

Proposition 7. The root $\alpha$ is the unique simple root not orthogonal to $\lambda$.

Proof. By Lemma $2, X^{*}(T)^{W_{s}}$ is freely generated by $\omega_{\alpha}$. Since $\lambda \in X^{*}(T)^{W_{s}}$ by Proposition 5, it follows that $\lambda=k \omega_{\alpha}$ for some non-zero $k \in \mathbb{Z}$. Also, we may write

$$
k \omega_{\alpha}=\lambda=\sum_{\beta \in \Pi} \lambda\left(h_{\beta}\right) \omega_{\beta}=\sum_{\beta \in \Pi} 2 \frac{(\lambda, \beta)}{(\beta, \beta)} \omega_{\beta} .
$$

Hence, for $\beta \in \Pi$, we have $(\lambda, \beta)=0$ if and only if $\beta \neq \alpha$.

Before continuing, we note the following implication of Proposition 7 for partial flag varieties in type $A$. 
Corollary 8. Suppose that $\mathrm{G}=\mathrm{SL}_{\mathrm{n}}(\mathbb{C})$ with $\mathrm{n} \geq 3$. There does not exist a partial flag variety $\mathrm{X}$ of $\mathrm{SL}_{\mathrm{n}}(\mathbb{C})$ with $\mathrm{b}_{2}(\mathrm{X})=1$ admitting an $\mathrm{SL}_{\mathrm{n}}(\mathbb{C})$-invariant contact structure. Equivalently, none of the Grassmannians $\operatorname{Gr}\left(k, \mathbb{C}^{n}\right), 1 \leq k \leq n-1$, supports an $\operatorname{SL}_{n}(\mathbb{C})$-invariant contact structure.

Proof. By Proposition 7 , the existence of such an $X$ would imply that there was a unique simple root not orthogonal to the highest root $\lambda$. However, for $G=\operatorname{SL}_{n}(\mathbb{C}), n \geq 3$, there are exactly two simple roots not orthogonal to $\lambda$. The formulation in terms of Grassmannians follows from their being the partial flag varieties of $S_{n}(\mathbb{C})$ having $b_{2}=1$.

Remark. Corollary 8 has an interesting consequence when $n$ is an even positive integer. Indeed, the odd-dimensional projective space $\mathbb{P}^{n-1}$ is then isomorphic to the projectivization of the minimal nilpotent orbit of $\mathrm{Sp}_{n}(\mathbb{C})$. In particular, $\mathbb{P}^{n-1}$ admits an $\mathrm{Sp}_{n}(\mathbb{C})$ invariant contact structure. Yet, Corollary 8 implies that this contact structure is not $\mathrm{SL}_{n}(\mathbb{C})$-invariant for $n \geq 4$.

Let us return to the matter at hand. Proposition 7 establishes that $S=\Pi \backslash\{\alpha\}$ is the collection of those simple roots which are orthogonal to $\lambda$, namely $S=\Lambda$. Hence, $G / P_{S}=$ $\mathrm{G} / \mathrm{P}_{\wedge}$, which is G-equivariantly isomorphic to $\mathbb{P}\left(\mathcal{O}_{\min }\right)$ via (4). It therefore remains to prove that (4) is additionally an isomorphism of contact varieties, recalling that $G / P_{S}=$ $\mathrm{G} / \mathrm{P}_{\wedge}$ has the $\mathrm{G}$-invariant contact structure $\mathbf{E} \subseteq \mathbf{T}_{\mathrm{G} / \mathrm{P}_{\wedge}}$ fixed in 3.3 .

Theorem 9. The map $\varphi: \mathrm{G} / \mathrm{P}_{\wedge} \rightarrow \mathbb{P}\left(\mathcal{O}_{\min }\right)$ in (4) is an isomorphism of contact varieties.

Proof. We are claiming that $\varphi^{*}\left(\mathbf{E}_{\min }\right)$ coincides with $\mathbf{E}$ when the former is regarded as a subbundle of $\mathbf{T}_{\mathrm{G} / \mathrm{P}_{\Lambda}}$. Observing that each of $\varphi^{*}\left(\mathbf{E}_{\min }\right)$ and $\mathbf{E}$ is a G-invariant corank-1

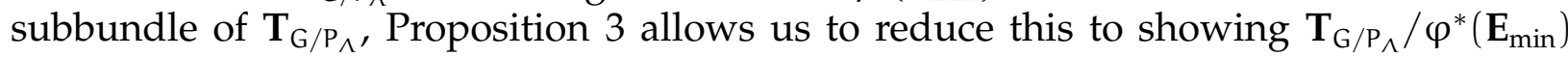
and $\mathbf{T}_{\mathrm{G} / \mathrm{P}_{\Lambda}} / \mathbf{E}$ to be isomorphic as holomorphic line bundles. The second line bundle is isomorphic to $\mathcal{L}(\lambda)$ by Proposition 5 , so we are further reduced to proving that the fibre $\left(\mathbf{T}_{\mathrm{G} / \mathrm{P}_{\Lambda}} / \varphi^{*}\left(\mathbf{E}_{\min }\right)\right)_{[\mathrm{e}]}$ has weight $\lambda$ as a T-representation.

Let $d_{[e]} \varphi:\left(T_{G / P_{\Lambda}}\right)_{[e]} \rightarrow\left(T_{\mathbb{P}\left(\mathcal{O}_{\min }\right)}\right)_{[\xi]}$ (where $\left.[\xi]=\varphi([e])\right)$ be the differential of $\varphi$ at $[e]$. Since $\varphi$ is T-equivariant, $\mathrm{d}_{[e]} \varphi$ is an isomorphism of T-representations. Furthermore, $\mathrm{d}_{[e]} \varphi\left(\varphi^{*}\left(\mathbf{E}_{\min }\right)_{[e]}\right)=\left(\mathbf{E}_{\min }\right)_{[\xi]}$, so that $\left(\mathbf{T}_{\mathrm{G} / \mathrm{P}_{\Lambda}} / \varphi^{*}\left(\mathbf{E}_{\min }\right)\right)_{[e]}$ and $\left(\mathrm{T}_{\mathbb{P}\left(\mathcal{O}_{\min }\right)}\right)_{[\xi]} /\left(\mathbf{E}_{\min }\right)_{[\xi]}$ are isomorphic T-representations. We also have an isomorphism $\left(T_{\mathbb{P}\left(\mathcal{O}_{\min }\right)}\right)_{[\xi]} \cong \mathfrak{g} / \mathfrak{p} \wedge$ from Section 3.2. under which $\left(\mathbf{E}_{\min }\right)_{[\xi]}$ identifies with $\left(\mathfrak{g}_{-\lambda}\right)^{\perp} / \mathfrak{p}_{\Lambda}$. Putting everything together, we have

$$
\left(\mathbf{T}_{\mathrm{G} / \mathrm{P}_{\Lambda}} / \varphi^{*}\left(\mathbf{E}_{\min }\right)\right)_{[e]} \cong\left(\mathrm{T}_{\mathbb{P}\left(\mathcal{O}_{\min }\right)}\right)_{[\xi]} /\left(\mathbf{E}_{\min }\right)_{[\xi]} \cong \mathfrak{g} /\left(\mathfrak{g}_{-\lambda}\right)^{\perp} .
$$

Since we have

$$
\left(\mathfrak{g}_{-\lambda}\right)^{\perp}=\mathfrak{b} \oplus \bigoplus_{\beta \in \Delta^{+} \backslash\{\lambda\}} \mathfrak{g}_{\beta},
$$

(10) implies that $\left(\mathbf{T}_{\mathrm{G} / \mathrm{P}_{\Lambda}} / \varphi^{*}\left(\mathbf{E}_{\mathrm{min}}\right)\right)_{[e]}$ is indeed the 1-dimensional T-representation of weight $\lambda$.

4.2. Example: The Grassmannian of Isotropic 2-Planes in $\mathbb{C}^{2 n}$. We now describe a class of explicit examples that satisfy the hypotheses of Theorem 1. To this end, let us set $\mathrm{G}=\mathrm{SO}_{2 n}(\mathbb{C})$ with $n \geq 4$. Given $\theta \in \mathbb{R} /(2 \pi \mathbb{Z})$, consider the $2 \times 2$ matrix

$$
R(\theta):=\left[\begin{array}{cc}
\cos (\theta) & -\sin (\theta) \\
\sin (\theta) & \cos (\theta)
\end{array}\right] .
$$


For $\theta_{1}, \theta_{2}, \ldots, \theta_{n} \in \mathbb{R} /(2 \pi \mathbb{Z})$, we define $R\left(\theta_{1}, \theta_{2}, \ldots, \theta_{n}\right)$ to be the $2 n \times 2 n$ block-diagonal matrix $R\left(\theta_{1}\right) \oplus R\left(\theta_{2}\right) \oplus \cdots \oplus R\left(\theta_{n}\right)$. Note that the $R\left(\theta_{1}, \theta_{2}, \ldots, \theta_{n}\right)$ constitute a maximal torus of the compact real form $\mathrm{SO}(2 \mathrm{n}) \subseteq \mathrm{SO}_{2 n}(\mathbb{C})$. Let $\mathrm{T} \subseteq \mathrm{SO}_{2 \mathrm{n}}(\mathbb{C})$ be the complexification of this maximal torus. We then choose our collection of simple roots to be $\Pi:=\left\{\alpha_{1}, \alpha_{2}, \ldots, \alpha_{n}\right\}$, where $\alpha_{j}: T \rightarrow \mathbb{C}^{*}$ is defined by the property

$$
\alpha_{j}\left(R\left(\theta_{1}, \theta_{2}, \ldots, \theta_{n}\right)\right)=e^{i\left(\theta_{j}-\theta_{j+1}\right)}
$$

for $j \in\{1, \ldots, n-1\}$, while $\alpha_{n}: T \rightarrow \mathbb{C}^{*}$ satisfies

$$
\alpha_{n}\left(R\left(\theta_{1}, \theta_{2}, \ldots, \theta_{n}\right)\right)=e^{i\left(\theta_{n-1}+\theta_{n}\right)} .
$$

The highest root $\lambda$ is then given by

$$
\lambda\left(R\left(\theta_{1}, \theta_{2}, \ldots, \theta_{n}\right)\right)=e^{i\left(\theta_{1}+\theta_{2}\right)} .
$$

Furthermore, the subset of simple roots orthogonal to $\lambda$ is $\Lambda=\Pi \backslash\left\{\alpha_{2}\right\}$.

Now, let $B: \mathbb{C}^{2 n} \otimes \mathbb{C}^{2 n} \rightarrow \mathbb{C}$ be the complexification of the dot product on $\mathbb{R}^{2 n}$. One then has the Grassmannian of isotropic 2-planes in $\mathbb{C}^{2 n}, \operatorname{Gr}_{B}\left(2, \mathbb{C}^{2 n}\right)$. More explicitly,

$$
\operatorname{Gr}_{\mathrm{B}}\left(2, \mathbb{C}^{2 \mathfrak{n}}\right):=\left\{\mathrm{V} \in \mathrm{Gr}\left(2, \mathbb{C}^{2 \mathrm{n}}\right): \mathrm{V} \subseteq \mathrm{V}^{\perp}\right\},
$$

where $V^{\perp}$ denotes the complement of $V \in G r\left(2, \mathbb{C}^{2 n}\right)$ with respect to $B$. This is a partial flag variety of $\mathrm{SO}_{2 n}(\mathbb{C})$, and one can verify that the $\mathrm{SO}_{2 n}(\mathbb{C})$-stabilizer of

$$
W:=\operatorname{span}\left\{e_{1}+i e_{2}, e_{3}+i e_{4}\right\} \in \operatorname{Gr}_{\mathrm{B}}\left(2, \mathbb{C}^{2 \mathrm{n}}\right)
$$

is precisely $\mathrm{P}_{\mathcal{\Lambda}} \subseteq \mathrm{SO}_{2 \mathrm{n}}(\mathbb{C})$. Hence, we have an $\mathrm{SO}_{2 \mathrm{n}}(\mathbb{C})$-equivariant variety isomorphism

$$
\mathrm{SO}_{2 \mathfrak{n}}(\mathbb{C}) / \mathrm{P}_{\Lambda} \cong \mathrm{Gr}_{\mathrm{B}}\left(2, \mathbb{C}^{2 \mathfrak{n}}\right) \text {. }
$$

By (4), we have another $\mathrm{SO}_{2 \mathfrak{n}}(\mathbb{C})$-equivariant isomorphism

$$
\mathbb{P}\left(\mathcal{O}_{\text {min }}\right) \cong \mathrm{Gr}_{\mathrm{B}}\left(2, \mathbb{C}^{2 \mathrm{n}}\right),
$$

where $\mathcal{O}_{\min }$ is the minimal nilpotent orbit of $\mathrm{SO}_{2 n}(\mathbb{C})$.

It remains to give the contact structure on $\mathrm{Gr}_{B}\left(2, \mathbb{C}^{2 \mathrm{n}}\right)$ for which (12) is an isomorphism of contact varieties. In other words, it remains to find the unique $\mathrm{SO}_{2 n}(\mathbb{C})$-invariant contact structure on $\mathrm{Gr}_{\mathrm{B}}\left(2, \mathbb{C}^{2 n}\right)$. To this end, let $\mathbf{F}$ denote the tautological bundle on $\mathrm{Gr}_{\mathrm{B}}\left(2, \mathbb{C}^{2 \mathfrak{n}}\right)$, whose fibre over $\mathrm{V} \in \mathrm{Gr}_{\mathrm{B}}\left(2, \mathbb{C}^{2 \mathfrak{n}}\right)$ is $\mathrm{V}$ itself. Note that $\mathbf{F}$ is a subbundle of the trivial bundle $\operatorname{Gr}_{B}\left(2, \mathbb{C}^{2 n}\right) \times \mathbb{C}^{2 n}$, so that we may consider the subbundle $\mathbf{F}^{\perp}$ of complements with respect to $\mathrm{B}$. By definition, $\mathbf{F} \subseteq \mathbf{F}^{\perp}$, and we may define

$$
\mathbf{E}:=\operatorname{Hom}\left(\mathbf{F}, \mathbf{F}^{\perp} / \mathbf{F}\right) \text {. }
$$

Note that $\mathbf{E}$ is canonically a subbundle of $\operatorname{Hom}\left(\mathbf{F}, \mathcal{O}^{\oplus 2 n} / \mathbf{F}\right)$, the pullback to $\mathrm{Gr}_{\mathrm{B}}\left(2, \mathbb{C}^{2 n}\right)$ of $\mathbf{T}_{\mathrm{Gr}\left(2, \mathbb{C}^{2 n}\right)}$. In fact, we have the inclusion

$$
\mathbf{E} \subseteq \mathbf{T}_{\mathrm{Gr}_{\mathrm{B}}\left(2, \mathbb{C}^{2 \mathfrak{n}}\right)}
$$

of subbundles of $\operatorname{Hom}\left(\mathbf{F}, \mathcal{O}^{\oplus 2 n} / \mathbf{F}\right)$, giving rise to a short exact sequence

$$
0 \rightarrow \mathbf{E} \rightarrow \mathbf{T}_{\mathrm{Gr}_{\mathrm{B}}\left(2, \mathbb{C}^{2 n}\right)} \rightarrow \wedge^{2}\left(\mathbf{F}^{\vee}\right) \rightarrow 0
$$

(see [6], Chapter 14). Since $\wedge^{2}\left(\mathbf{F}^{\vee}\right)=\operatorname{det}\left(\mathbf{F}^{\vee}\right)$ is a line bundle, E is a corank-1 subbundle of $\mathbf{T}_{\mathrm{Gr}_{\mathrm{B}}\left(2, \mathbb{C}^{2 n}\right)}$. Indeed, we have the following proposition. 
Proposition 10. The subbundle $\mathbf{E} \subseteq \mathbf{T}_{\mathrm{Gr}_{\mathrm{B}}\left(2, \mathbb{C}^{2 n}\right)}$ is the unique $\mathrm{SO}_{2 n}(\mathbb{C})$-invariant contact structure on $\mathrm{Gr}_{\mathrm{B}}\left(2, \mathbb{C}^{2 \mathrm{n}}\right)$.

Proof. By Proposition 3 and the discussion at the end of Section 2 , the $\mathrm{SO}_{2 \mathrm{n}}(\mathbb{C})$-invariant contact structure on $\operatorname{Gr}_{B}\left(2, \mathbb{C}^{2 n}\right)$ is the unique subbundle of $\mathbf{H} \subseteq \mathbf{T}_{\mathrm{Gr}_{\mathrm{B}}\left(2, \mathbb{C}^{2 n}\right)}$ such $\mathbf{H}$ is $\mathrm{SO}_{2 \mathrm{n}}(\mathbb{C})$-invariant and $\mathrm{T}_{\mathrm{Gr}_{\mathrm{B}}\left(2, \mathbb{C}^{2 n}\right)} / \mathbf{H}$ is the ample generator of $\operatorname{Pic}\left(\mathrm{Gr}_{\mathrm{B}}\left(2, \mathbb{C}^{2 \mathfrak{n}}\right)\right)$. Accordingly, it will suffice to prove that $\mathbf{E}$ possesses these two properties. For the former, note that $\mathbf{F}^{\perp} / \mathbf{F}$ is an $\mathrm{SO}_{2 \mathfrak{n}}(\mathbb{C})$-invariant subbundle of $\mathcal{O}^{\oplus 2 \mathfrak{n}} / \mathbf{F}$. Hence, $\mathbf{E}=\operatorname{Hom}\left(\mathbf{F}, \mathbf{F}^{\perp} / \mathbf{F}\right)$ is an $\mathrm{SO}_{2 n}(\mathbb{C})$-invariant subbundle of $\operatorname{Hom}\left(\mathbf{F}, \mathcal{O}^{\oplus 2 n} / \mathbf{F}\right)$, and therefore also of $\mathbf{T}_{\mathrm{Gr}_{B}\left(2, \mathbb{C}^{2 n}\right)}$. For our second property, (13) gives a bundle isomorphism

$$
\mathbf{T}_{\mathrm{Gr}_{\mathrm{B}}\left(2, \mathbb{C}^{2 n}\right)} / \mathbf{E} \cong \operatorname{det}\left(\mathbf{F}^{\vee}\right) .
$$

The bundle $\operatorname{det}\left(\mathbf{F}^{\vee}\right)$ is indeed the ample generator of $\operatorname{Pic}\left(\operatorname{Gr}_{\mathrm{B}}\left(2, \mathbb{C}^{2 n}\right)\right)$, so our proof is complete.

We wish to conclude with a comparison of our presentation of the $\mathrm{SO}_{2 n}(\mathbb{C})$-invariant contact structure on the isotropic Grassmannian to the one presented in [7] (pp.353-354), whose distribution we will denote by $\mathbf{P}$. There, $\operatorname{Gr}_{B}\left(2, \mathbb{C}^{2 \mathfrak{n}}\right)$ is given an alternative presentation, as a parameter space for lines in a hyperquadric of dimension $2 n-2$. If $\ell$ is line in the hyperquadric representating a point in the parameter space, and if we choose an isomorphism $\ell \cong \mathbb{P}^{1}$, the fibre $\mathbf{P}_{\ell}$ is the space of global holomorphic sections of the (2n-4)fold direct sum of the hyperplane bundle on the $\mathbb{P}^{1}$. One must choose an isomorphism for each point in order to describe $\mathbf{P}$ and so this description - while explicit - is local. Our presentation of the unique $\mathrm{SO}_{2 n}(\mathbb{C})$-invariant contact structure, with distribution $\mathbf{E}$ given above, does not depend on a family of isomorphisms and uses the tautological bundle on the isotropic Grassmannian directly.

\section{REFERENCES}

[1] Beauville, A. Fano contact manifolds and nilpotent orbits. Comment. Math. Helv. 73, 4 (1998), 566583.

[2] Bоотнву, W. M. Homogeneous complex contact manifolds. In Proc. Sympos. Pure Math., Vol. III. American Mathematical Society, Providence, R. I., 1961, pp. 144-154.

[3] Воотнву, W. M. A note on homogeneous complex contact manifolds. Proc. Amer. Math. Soc. 13 (1962), 276-280.

[4] BUCZYŃSKI, J. Duality and integrability on contact Fano manifolds. Doc. Math. 15 (2010), 821-841.

[5] Demailly, J.-P. On the Frobenius integrability of certain holomorphic p-forms. In Complex geometry (Göttingen, 2000). Springer, Berlin, 2002, pp. 93-98.

[6] Guillemin, V., AND SternberG, S. Variations on a Theme by Kepler, vol. 42 of American Mathematical Society Colloquium Publications. American Mathematical Society, Providence, RI, 1990.

[7] HWANG, J.-M. Geometry of minimal rational curves on Fano manifolds. In School on Vanishing Theorems and Effective Results in Algebraic Geometry (Trieste, 2000), vol. 6 of ICTP Lect. Notes. Abdus Salam Int. Cent. Theoret. Phys., Trieste, 2001, pp. 335-393.

[8] KebeKUS, S. Lines on contact manifolds. J. Reine Angew. Math. 539 (2001), 167-177.

[9] Kebekus, S., Peternell, T., Sommese, A. J., AND WiśnieWsKi, J. A. Projective contact manifolds. Invent. Math. 142, 1 (2000), 1-15.

[10] KoBAyASHI, S., AND OCHIAI, T. Characterizations of complex projective spaces and hyperquadrics. J. Math. Kyoto Univ. 13 (1973), 31-47. 
[11] KOlláR, J. Rational Curves on Algebraic Varieties, vol. 32 of Ergebnisse der Mathematik und ihrer Grenzgebiete. 3. Folge. A Series of Modern Surveys in Mathematics [Results in Mathematics and Related Areas. 3rd Series. A Series of Modern Surveys in Mathematics]. Springer-Verlag, Berlin, 1996.

[12] LEBRUN, C., AND SALAMON, S. Strong rigidity of positive quaternion-Kähler manifolds. Invent. Math. 118, 1 (1994), 109-132.

[13] Peternell, T. Contact structures, rational curves and Mori theory. In European Congress of Mathematics, Vol. I (Barcelona, 2000), vol. 201 of Progr. Math. Birkhäuser, Basel, 2001, pp. 509-518.

[14] Peternell, T. Subsheaves in the tangent bundle: integrability, stability and positivity. In School on Vanishing Theorems and Effective Results in Algebraic Geometry (Trieste, 2000), vol. 6 of ICTP Lect. Notes. Abdus Salam Int. Cent. Theoret. Phys., Trieste, 2001, pp. 285-334.

[15] SNOW, D. M. Homogeneous vector bundles. In Group Actions and Invariant Theory (Montreal, PQ, 1988), vol. 10 of CMS Conf. Proc. Amer. Math. Soc., Providence, RI, 1989, pp. 193-205.

Department of MATHEMATICS, University of TORONTO, CANADA

E-mail address: peter.crooks@utoronto.ca, stever@math.toronto.edu 